\title{
Epilepsia e depressão: abordagem na atenção primária como estratégia de saúde pública
}

\author{
Epilepsy and depression: concomitant approach as a public health strategy
}

Epilepsia y depresión: un enfoque de atención primaria como estrategia de salud pública.

Marcelo Pereira Cintra ${ }^{1 \star}$, Chan Tiel Yuen ${ }^{1}$.

\section{RESUMO}

Objetivo: Discutir aspectos significativos do manejo da epilepsia, associada à estados depressivos na atenção primária de saúde. Revisão bibliográfica: A epilepsia é uma afecção que repercute na qualidade de vida dos pacientes, sendo frequentemente associada à transtornos do humor, especialmente à depressão. No modelo adotado no sistema de saúde pública no Brasil com viés generalista, os pacientes com epilepsia também são atendidos primordialmente pelos serviços de atenção primária e a condução destes doentes acaba enfrentando obstáculos tanto no diagnóstico como no tratamento. A adoção de proposições já experimentadas anteriormente servem de referência para os diversos serviços de atenção primária permitindo uma oferta superior de cuidados de que carecem estes pacientes, bem como diminuir os ônus derivados da conduta inadequada observada frequentemente na prática neuropsiquiátrica por profissionais sem especialização. Considerações finais: Apesar da complexidade que envolve o assunto, já existem protocolos que permitem uma condução clínica adequada com redução de morbidades, melhora na qualidade de vida e redução nos custos implícitos decorrentes do surgimento associado destas entidades neuropsiquiátricas, especialmente na saúde pública do Brasil.

Palavras-chave: Epilepsia, Depressão, Transtornos mentais, Saúde pública.

\begin{abstract}
Objective: To discuss significant aspects of the management of epilepsy associated with depressive states in primary health care. Bibliographic review: Epilepsy is a condition that affects the quality of life of patients and is often associated with mood disorders, especially depression. In the model adopted in the public health system in Brazil with a generalist bias, patients with epilepsy are also primarily cared for by primary care services and the management of these patients ends up facing obstacles both in diagnosis and in treatment. The adoption of previously tried propositions serve as a reference for the various primary care services, allowing for a superior offer of care that these patients need, as well as reducing the burden arising from the inappropriate conduct frequently observed in neuropsychiatric practice by professionals without specialization. Final considerations: Despite the complexity involved in the subject, there are already protocols that allow for an adequate clinical management with a reduction in morbidities, improvement in quality of life and reduction in implicit costs resulting from the associated emergence of these neuropsychiatric entities, especially in public health in Brazil.
\end{abstract}

Keywords: Epilepsy, Depression, Mental disorders, Public health.

1 Universidade de Vassouras (UV), Vassouras - RJ.

*E-mail: marcelopcintra18@outlook.com

SUBMETIDO EM: 9/2021

ACEITO EM: 9/2021

PUBLICADO EM: 10/2021 


\section{RESUMEN}

Objetivo: discutir aspectos significativos del manejo de la epilepsia asociada a estados depresivos en la atención primaria de salud. Revisión bibliográfica: La epilepsia es una afección que afecta la calidad de vida de los pacientes y, a menudo, se asocia con trastornos del estado de ánimo, especialmente depresión. En el modelo adoptado en el sistema público de salud en Brasil con un sesgo generalista, los pacientes con epilepsia también son atendidos principalmente por los servicios de atención primaria y el manejo de estos pacientes termina enfrentando obstáculos tanto en el diagnóstico como en el tratamiento. La adopción de propuestas previamente probadas sirve de referencia para los distintos servicios de atención primaria, permitiendo una oferta superior de atención que necesitan estos pacientes, así como la reducción de la carga derivada de las conductas inapropiadas frecuentemente observadas en la práctica neuropsiquiátrica por profesionales sin especialización. Consideraciones finales: A pesar de la complejidad que implica el tema, ya existen protocolos que permiten un adecuado manejo clínico con reducción de morbilidades, mejora de la calidad de vida y reducción de los costes implícitos derivados de la aparición asociada de estas entidades neuropsiquiátricas, especialmente en salud pública en Brasil.

Palabras clave: Epilepsia, Depresión, Trastornos mentales, Salud pública.

\section{INTRODUÇÃO}

A epilepsia deve ser compreendida como uma alteração transitória decorrente de funcionamento neuronal anormal, cursando com hiperatividade e descargas elétricas cerebrais, onde estejam exclusas causas como infecções agudas, uso de drogas, trauma cranioencefálico ou alterações do metabolismo (COSTA LLO, et al., 2020). A crise epilética é manifestação extremamente expressiva, caracterizada pelo surgimento abrupto de sintomas, especialmente, motores, autonômicos e de consciência, mas que envolvem outros espectros de alterações, relacionadas ao sítio anatômico onde se apresentam as descargas que modificam a dinâmica cerebral. Embora se reconheça a relevância destas manifestações, e seus impactos na vida dos pacientes, os demais contextos em que a doença se apresenta são tão relevantes, causando também extremo sofrimento (AMERICAN PSYCHIATRIC ASSOCIATION (APA), 2014; FAVARO VF, 2019).

Dentre as afecções que acompanham os pacientes portadores de epilepsia, os distúrbios psiquiátricos são os mais recorrentes e dentre estes temos a depressão, sendo a ocorrência deste transtorno psiquiátrico, mais elevado, quando comparado ao que se observa no restante da população (KANNER AM e BALABANOV A, 2002). A prevalência de transtorno depressivo maior, durante a vida, foi estimado em $9,4 \%$ da população geral na idade adulta, mas estudos recentes avaliaram sua simultaneidade em mais de $23 \%$ nos pacientes com epilepsia, tornando mandatória a compreensão dos mecanismos que determinam este aumento expressivo na concorrência destes estados (ELGER CE, et al., 2016; SCOTT AJ, et al., 2017).

Os transtornos do humor englobam um grupo significativo de doenças psiquiátricas, dentre estas temos os transtornos depressivos. Estes trazem como principais características a persistência de um estado de humor melancólico e irritadiço, acompanhado de manifestações somáticas e alterações intelectuais que impactam expressivamente na qualidade de vida dos pacientes. A depressão enquadra-se dentro deste extenso conjunto de patologias designadas como transtornos depressivos (APA, 2014).

Estudos têm acrescentado evidências do nexo direcionado entre epilepsia e distúrbios psiquiátricos, com fortes indicativos de seus mecanismos fisiopatológicos. Diversos mecanismos patogênicos que envolvem crises epilépticas e depressão ainda estão em permanente avaliação para que seja estabelecida a exata correlação clínica entre eles, demonstrando como a ansiedade e os transtornos depressivos ocorrem em um nível mais elevado nos pacientes com epilepsia. Os fortes indícios de que o surgimento de estados depressivos sucede a ocorrência das crises ainda precisam de maior esclarecimento (SCOTT AJ, et al., 2017).

Malgrado o reconhecimento da fisiopatologia associada ao surgimento de depressão em pacientes epiléticos, o diagnóstico concomitante destas duas afecções é ainda sistematicamente negligenciado em atendimento 
clínico não especializado, havendo uma recorrência em relevar sintomas depressivos e atribuí-los unicamente como derivados dos eventos convulsionais ou reativos ao tratamento medicamentoso (KANNER AM e BALABANOV A, 2002).

Muito se discute na cátedra neuropsiquiátrica sobre fatores que se apresentam fortemente vinculados à relação destas duas enfermidades, além de buscar protocolos que ajam em consonância tanto para o diagnóstico quanto ao tratamento. Apesar das lacunas existentes no conhecimento desta patogênese e das atipias nas apresentações clínicas, é implausível que os pacientes acometidos permaneçam sem um tratamento adequado às suas necessidades e à obtenção de melhores resultados terapêuticos (KANNER AM e BALABANOV A, 2002).

Em 1977, foi lançada a campanha Bringing Epilepsy "Out of the Shadows" uma iniciativa conjunta da Organização Mundial da Saúde (OMS), da Liga Internacional contra Epilepsia (ILAE) e do Escritório Internacional para a epilepsia (IBE), com escopo literal de "trazer epilepsia para Fora das Sombras", buscando dentre outros objetivos, melhorias no diagnóstico, tratamento, prevenção e inclusão social do transtorno epiléptico em todo o mundo (OMS, 2001). No Brasil, a ILAE tem uma representação que desenvolve os seus preceitos, sendo mediada pela Liga Brasileira de Epilepsia (LBE) com missão contínua de incentivo ao ensino e pesquisa com fulcro na melhoria diagnóstica e terapêutica da epilepsia de uma forma mais abrangente (LBE, 2020).

O objetivo do presente estudo é discutir aspectos significativos do manejo da epilepsia, quando associada à estados depressivos, com intuito de expandir o conhecimento sobre o problema, apresentando abordagens, que possibilitam às diversas especialidades, que lidam com esta questão na atenção primária, uma orientação mais adequada destes pacientes, especialmente na saúde pública do Brasil.

\section{REVISÃO BIBLIOGRÁFICA}

Nos países em desenvolvimento foram observadas falhas no tratamento da epilepsia, decorrentes de desigualdades econômicas no atendimento em saúde, associadas à persistência de uma percepção de estigma social por parte dos pacientes, contribuindo para níveis insatisfatórios de adesão aos serviços de saúde. Esta deficiência é especialmente elevada na atenção primária, predispondo à ocorrência de níveis insatisfatórios tanto de procura ao tratamento por parte dos pacientes, como ao correto reconhecimento de manifestações psiquiátricas clínicas, associadas à epilepsia, tal como a depressão (KEDE J, et al., 2008).

A correção desta conjuntura constitui-se em aspecto indispensável para melhoria, sendo congruente com a acepção de melhoria da qualidade de vida destes pacientes, ao possibilitar opções terapêuticas e apoio multidisciplinar apropriado. Contudo, resiste ainda certo obstáculo à instituição de uma linha de ação terapêutica a esses pacientes (KEDE J, et al., 2008).

As manifestações psiquiátricas subjacentes à epilepsia, como os transtornos depressivos, também sofrem deste tipo de lapso, tendo como principais origens, a propensão dos clínicos em minimizar os sintomas depressivos e da própria dificuldade em identificar muitas das manifestações atípicas que acompanham estes pacientes. Outro aspecto que interfere neste prejuízo ao tratamento é a indisposição dos pacientes em demonstrar queixas depressivas por medo de estigmatização. Finalmente, a percepção errônea de que todas as drogas antidepressivas diminuem o limiar convulsivo, o que determina certo grau de oposição à prescrição deste tipo de fármacos (KANNER AM e BALABANOV A, 2002).

A despeito de ainda subsistir alguma divergência na literatura, no que concerne a relação de concorrência destas duas entidades (WALSH S, et al., 2018). Diversas observações demonstraram a concomitância de epilepsia e depressão, constituindo-se a depressão como a alteração psiquiátrica mais comum nos pacientes com epilépticos (SCOTT RA, et al., 2001). Blaszczyk B e Czuczwar SJ (2016) mensuraram, em seu estudo, uma taxa de prevalência de transtornos do humor entre os pacientes com epilepsia 4 a 5 vezes maior do que na população em geral.

Em atenção à elevada incidência da epilepsia, sobretudo nos países em desenvolvimento, houve um aumento na realização de estudos acerca do tema, havendo uma consolidação no entendimento dos 
mecanismos envolvidos na gênese da epilepsia e de suas complicações (GASPARINI CS, 2016). A contribuição destas pesquisas abriu um novo caminho na oferta de tratamento com abordagens que envolvem a uma intervenção amparada de longo prazo, sendo uma referência basilar em qualquer programa de tratamento nesta área (HOPKER C, et al., 2017;).

Estudos sobre a fisiopatologia, envolvendo crises epilépticas acompanhadas de estados depressivos, demonstraram que a depressão surge frequentemente associada à epilepsia do lobo temporal (BLASZCZYK B e CZUCZWAR SJ, 2016; ELGER CE, et al., 2016). Existem diversos mecanismos influenciando a concorrência de epilepsia e depressão, alterações anatômicas envolvendo hipocampo, distúrbios nos sistemas de neurotransmissores, alterações imunológicas e fatores genéticos, dentre outros (BLASZCZYK B e CZUCZWAR SJ, 2016).

Aduz-se que a secreção e metabolismo de neurotransmissores, em seus sítios de ação, estão intensamente envolvidos no processo de desencadeamento das convulsões e, concorrentemente, de estados depressivos, indicando uma origem genética endógena neste quadro disfuncional (KANNER AM e BALABANOV A, 2002; PÉREZ EB, 2015). Há consenso de que o neurotransmissor glutamato tem ação decisiva no desenvolvimento de crises epilépticas, havendo sua atuação também no desenvolvimento de depressão, apesar de ainda não ter esclarecidos seus mecanismos na gênese dos estados depressivos (BLASZCZYK B e CZUCZWAR SJ, 2016).

Cabe ressaltar que a baixa efetividade nas intervenções nesses pacientes epilépticos, em vigência de depressão, procede também da noção de que o emprego de drogas antiepiléticas estabelece condição determinante no desencadeamento de alterações de humor. Não se pode, contudo, relevar a influência dessas substâncias com tropismo pelos receptores GABAérgicos, tais como o valproato de sódio, topiramato, dentre outros, no incremento de sintomas depressores (PÉREZ EB, 2015). Apesar desta interação indesejada, estudos indicam que a associação de antidepressivos concomitantemente às drogas antiepiléticas permitem um melhor resultado nesses pacientes (KANNER AM e BALABANOV A, 2002).

Apesar disso, observa-se ainda certa resistência à instituição de uma linha de ação terapêutica a esses pacientes por parte daqueles sem um maior aprofundamento no assunto. $O$ fato de que certas medicações antidepressivas possuem capacidade de reduzir o limiar convulsivante representa a condição típica do problema. Tornando forçoso que as condutas de tratamento medicamentoso, dos transtornos do humor associados à epilepsia, ainda estejam sujeitas profundamente à prática de especialistas, apesar do atendimento destes profissionais não sejam frequentemente acessíveis (KEDE J, et al., 2008; KANNER AM, 2016).

Recentemente, estudos introduziram novos entendimentos sobre este tema, reafirmando compreensão anterior sobre a segurança no uso conjunto de antidepressivos e medicações anticrise (XIANGMIAO Q, et al. 2018). A adoção de medicamentos psiquiátricos do grupo dos inibidores da receptação de serotonina demonstrou-se segura em sua utilização nesses pacientes, tais como, fluoxetina, sertralina, paroxetina, dentre outros do mesmo grupo (ELGER CE, et al., 2016; XIANGMIAO Q, et al. 2018).

Observa-se diante do discutido até o momento, que a lacuna no tratamento destes pacientes não está restrita a um aspecto em particular, mas decorre de condicionantes econômicos, políticos, culturais e estruturais das sociedades, bem como da dinâmica da própria história natural da patologia e de seus desdobramentos. Não obstante sua relevância como entidade nosológica e da existência de tratamentos eficazes, a epilepsia e suas comorbidades associadas não estão elencadas como prioridade absoluta de saúde pública (SCOTT AJ, et al., 2021).

Scott RA, et al. (2001) apresentaram em seu estudo, os principais fatores que corroboram esta perspectiva em serviços públicos de saúde, não só no Brasil, mas como em grande parte do mundo em desenvolvimento. Os autores mencionam a destinação orçamentária abaixo das necessidades da população, como fator fundamental para ineficiência deste tipo de abordagem terapêutica. Expõem ainda, a visão predominante entre os gestores, que favorece a aplicação de recursos em áreas consideradas prioritárias, tais como as doenças infeciosas. 
Outras dimensões apresentadas são, a falta de infraestrutura específica para este tipo de tratamento, ausência de programas assistenciais direcionados às especificidades de cada paciente, ausência de estudos demonstrando a importância destas políticas, dentre outras. Sustentam a importância do investimento em estudos que forneçam dados que demostrem a relação custo-benefício de tratar a epilepsia, estes seriam de valor inestimável para convencer os planejadores de saúde da necessidade priorizar o tratamento da epilepsia. Kede J, et al. (2008) acrescentam que preparo específico para os profissionais generalistas e equipe de enfermagem seria outra dimensão importante na melhoria do atendimento.

A análise epidemiológica da epilepsia, em nosso país, ainda carece de um maior aprofundamento, no entanto, estudos demonstraram uma variabilidade acentuada de prevalência da afecção de acordo com diversas regiões do país. Na Grande São Paulo foi encontrada uma prevalência situada em torno de 11,9 por 1.000 habitantes, já no Rio Grande do Sul foi encontrada um valor mais elevado da ordem de 16,5 por 1.000 habitantes (NOLASCO MN, et al., 2020). A transposição destes resultados para todo o Brasil denota uma alta ocorrência da patologia, justificando incluí-la como um grave problema de saúde pública. Acresce gravidade à questão, a constatação de que ausência de uma terapêutica adequada, acaba por tornar o paciente inabilitado ao trabalho e consequentemente, determina um ônus significativo à família, mas que atinge também toda a sociedade (GASPARINI CS, 2016).

A falta de uma visão de Estado na condução de políticas de saúde acaba determinando inércia ou ações ineficazes, que invariavelmente são conexas à incapacidade de percepção das reais necessidades a serem implementadas no enfrentamento de diversos agravos à saúde, sendo este problema recursivo em países em desenvolvimento. Tradições culturais, prioridades ambíguas, carências na infraestrutura do sistema de saúde, a adoção de condutas indevidas e de fornecimento inadequado de medicamentos antiepilépticos contribuem para dificultar o tratamento e influenciam no resultado das estratégias de oferta de serviços de saúde aos indivíduos (KEDE J, et al., 2008; SCOTT RA, et al., 2001).

No caso brasileiro, há todo um arcabouço jurídico com objetivo de atender as demandas destes pacientes neurológicos, existem ainda projetos que estabeleceram novas metodologias ao introduzirem um novo olhar sobre a condução dos pacientes epilépticos, através do sistema de atendimento primário à saúde já implantado no SUS (Sistema Único de Saúde). Tal perspectiva conta com a atuação conjunta da comunidade, buscando propagar um modelo de tratamento da epilepsia de longo prazo e integral para utilização em âmbito nacional, tendo a rede primária de saúde como principal via de disseminação de serviços. Apesar destes projetos não contemplarem especificamente as comorbidades, sem dúvida contribuem para uma melhor disposição de toda problemática (MIN LL e SANDER JWAS, 2003; MIN LL, et al., 2007).

Neste sentido foi expedida a Portaria ํㅜ 1.161/2005 SAS/MS, que instituiu a Política Nacional de Atenção ao Portador de Doenças Neurológicas, imbuída de implementar a intervenção precoce, com ênfase em ações de promoção e prevenção de saúde. Sua consignação buscou estruturar a rede de serviços e de cuidados a esses pacientes, tendo em conta reduzir agravos e aperfeiçoar o acesso dos pacientes ao atendimento especializado. Este instrumento jurídico estabeleceu a criação da Câmara Técnica, subordinada à Secretaria de Atenção à Saúde (SAS/MS), com o objetivo de monitorar a implantação da política instituída e para as diligências necessárias à sua organização, sobretudo através de portarias específicas. Neste âmbito, foi exarada a Portaria SAS/MS no 1319, de 25 de novembro de 2013, retificada em 27 de novembro de 2015 e que revogou instrumentos anteriores que instituiu protocolos e diretrizes terapêuticas para epilepsia a serem adotados no SUS (MINISTÉRIO DA SAÚDE, 2013).

Tendo em vista a situação crítica dos pacientes portadores de epilepsia no Brasil e em adesão à campanha global liderada pela Organização Mundial da Saúde (OMS), Liga Internacional Contra Epilepsia (ILAE), foram constituídas propostas para serem empregadas no fomento de programas públicos para o tratamento desta doença em todo o mundo em desenvolvimento. No Brasil, o Projeto Assistência à Saúde de Pacientes com Epilepsia (ASPE) é o braço executivo dessa campanha com um projeto de educação permanente, reconhecido internacionalmente, cujos esforços principais foram o estudo epidemiológico da epilepsia, a capacitação de profissionais para o manejo integral de pacientes epilépticos e para compartilhamento de conhecimento e expansão do projeto a nível nacional (KEDE J, et al., 2008; MIN LL e SANDER JWAS, 2003). 
Projeto piloto foi implementado no Estado de São Paulo, e foram estabelecidos, de forma preliminar, critérios de avaliação do projeto que objetivavam consubstanciar seus resultados, por intermédio da mensuração dos seguintes requisitos apresentados em seguida. Primeiramente, a questão da lacuna de tratamento com demonstração de redução do número de pacientes não tratados. Em segundo plano, a eficácia clínica da intervenção com avaliação do número de pacientes sem crises ou com melhora expressiva das crises, associada à baixa desistência ao tratamento em decorrência aos efeitos colaterais do tratamento conferindo segurança e tolerabilidade ao mesmo, e em virtude da observação de mudanças sociais observadas em pacientes e na comunidade. Em terceiro lugar, a eficiência do programa de treinamento em produzir alterações na conduta das equipes de saúde avaliada por meio do emprego de questionários. Finalmente, a eficácia do programa em diminuir o ônus social e econômico da epilepsia medida pela redução no absenteísmo na escola e trabalho, bem como por meio do número de pacientes que retornam à escola ou conseguem emprego (MIN LL, et al., 2007).

As informações colhidas no estudo demonstraram resultados encorajadores, inclusive apresentando aspectos que merecem atenção dos gestores como fatores críticos de desempenho das unidades de saúde, onde se pretendem implantar modelos semelhantes. Houve efetivo resultado no controle de convulsões, a percepção subjetiva das equipes de saúde e os pacientes e parentes foram fortemente consoantes em todos os aspectos. A desistência aos tratamentos em decorrência de efeitos colaterais foi mínima, bem como à intolerância medicamentosa. O resultado social foi positivo ao ser observada redução do absenteísmo na escola e no trabalho, sendo que neste último aspecto o resultado não foi tão expressivo ao não haver uma participação ativa de empresas no processo (MIN LL e SANDER JWAS, 2003).

A OMS analisando experiências internacionais e investigando critérios indispensáveis à constituição de serviços de atendimento dos pacientes com epilepsia, corrobora a perspectiva de condução otimizada desta patologia e de suas intercorrências também na atenção básica. $O$ estudo suscitou a elaboração de modelos de atendimento em epilepsia com enfoque das seguintes perspectivas: associação da atenção primária com a secundária na orientação clínica dos pacientes; definição nítida do papel do especialista e da clínica geral; clínicas de referência com enfoque em pacientes epilépticos na atenção secundária, dentre outras (ORGANIZAÇÃO MUNDIAL DE SAÚDE (OMS), 2018).

O atendimento deste tipo de paciente, nas condições atualmente dispostas nos serviços de saúde no Brasil, ainda está aquém da medicina justa e igualitária que busca nosso sistema de saúde. Para isso é necessária uma mudança de paradigma com a integração de serviços de maior complexidade, aos serviços de atenção básica, pelas qualidades que estes apresentam de maior cobertura de atendimento nas comunidades e por melhor resultado quando comparado seu custo diante da efetividade (KEDE J, et al., 2008).

Ainda permanece um hiato de formação do médico de família, concernente à epilepsia, impedindo um melhor controle desses pacientes. O especialista em neurologia, até mesmo em virtude de sua escassez, não pode ser encarregado da condução exclusiva destes pacientes. A proposição do Projeto Assistência à Saúde de Pacientes com Epilepsia (ASPE) demonstrou-se viável ao conjugar os recursos de forma racional e a estimular capacitação formal da equipe de profissionais das unidades básicas de saúde (KEDE J, et al., 2008; MIN LL, et al., 2007).

A adoção e implantação destas proposições são notadamente importantes em virtude do modelo de atenção da saúde pública brasileira, visto que a dimensão de atendimento descentralizada e hierarquizada, em consequência de sua heterogeneidade, nem sempre propicia os meios necessários ao cumprimento da política nacional de atenção aos portadores de doenças neurológicas. Componentes essenciais desta política têm se constituído como entraves na estruturação dessas redes de atendimento, mas apresentam ao mesmo tempo propriedades que podem permitir harmonizar o fornecimento de cuidados integrais que necessitam estes pacientes, bem como reduzir os encargos consequentes da abordagem inadequada observada frequentemente na prática neuropsiquiátrica (CONASS, 2015; KEDE J, et. al., 2008). 


\section{CONSIDERAÇÕES FINAIS}

Este estudo veio expor evidências sobre a confluência de estados depressivos em pacientes epilépticos e os óbices enfrentados na condução desses pacientes, em especial, nos serviços de saúde pública. Conjuntamente, apresentou prática implantada no país, como forma mitigar as deficiências encontradas, assim como reduzir os custos implícitos na gestão inadequada desta condição clínica. O modelo aplicado apresentou resultados promissores, revelando-se viável. Ao investir na capacitação do médico da atenção básica, associar conformação diagnóstica e terapêutica, integração de apoio psicológico e participação da comunidade local, o modelo confirma-se como oportunidade de melhoria dos serviços de saúde, devendo ser sua implantação estimulada, para que se possibilite a entrega de cuidados mais adequados a esses casos.

\section{REFERÊNCIAS}

1. AMERICAN PSYSHIATRIC ASSOCIATION. APA. Manual Diagnóstico e Estatístico de Transtornos Mentais - DSM5. 5.ed. Porto Alegre: Artmed, 2014; p.155-157.

2. BLASZCZYK B, CZUCZWAR SJ. Epilepsy coexisting with depression. Pharmacological Reports. 2016; 68(5): 108492.

3. CONASS. Para entender a gestão do SUS - Assistência de média e alta complexidade. ed. Brasília: CONASS. 2015; 4(20): 129-136.

4. COSTA LLO, et al. Atualização em epilepsia: revisão de literatura. Revista de Medicina. 2020. 99(2):170-181.

5. ELGER CE, et al. "Diagnosing and treating depression in epilepsy", Seizure. 2017; 44: 184-193.

6. FAVARO VF. Depressão, ansiedade interictal e suicídio. In: Ferreira AG, et al. Manual prático de neuropsiquiatria de epilepsia. 1.ed. Rio de janeiro: Elsevier; 2019; p.145-154.

7. GASPARINI CS. A incidência da epilepsia em uma cidade de porte médio: estudo em arquivos médicos informatizados e prontuários. Botucatu, SP. Dissertação (Mestrado em Medicina) - Faculdade de Medicina de Botucatu. Universidade Estadual Paulista Júlio de Mesquita Filho, Botucatu, 2016; 59 p.

8. HOPKER, CD, et al. "The individual with epilepsy: perceptions about the disease and implications on quality of life", CoDAS, 2017; 29(1):1-8.

9. KANNER AM, BALABANOV A. Depression and epilepsy: how closely related are they? Neurology 2002; 58(8 Suppl 5): S27-39.

10. KANNER, AM. Management of psychiatric and neurological comorbidities in epilepsy. Nat Rev Neurol 12, $2016 ; 12$ : 106-116.

11. KEDE J, et al. Atenção primária à saúde e epilepsia: Revisão de literatura. Journal os Epilepsy and Clinical Neurophysiology. 2008; 14(4):177-83.

12. LIGA BRASILEIRA DE EPILEPSIA (LBE). História: A Liga Brasileira de Epilepsia nos seus 70 anos. São Paulo: LBE. Disponível em: https://www.epilepsia.org.br/historia. Acessado em: 28 de dezembro de 2020.

13. MINISTÉRIO DA SAÚDE. Portaria SAS/MS n 1319, de 25 de novembro de 2013. Retificada em 27 de novembro de 2015 Revoga a Portaria no 492/SAS/MS, de 23 de setembro de 2010. 2015;8(1):2-25. Disponível em: http://portalarquivos.saude.gov.br/images/pdf/2016/fevereiro/04/Epilepsia---PCDT-Formatado--.pdf. Acessado em: 22 de janeiro de 2021.

14. MIN LL, SANDER JWAS. National demonstration project on epilepsy in Brazil. Arquivos de Neuropsiquiatria. 2003; 61(1): 153-6.

15. MIN LL, et al. Demonstration project on epilepsy in Brazil: Outcome assessment. Arquivos de Neuropsiquiatria. 2007; 65(SUPPL. 1): 58-62.

16. NOLASCO MN, et al. Epidemiologia dos casos de internação hospitalar por epilepsia no estado do Tocantins em 2018. Brazilian Journal of Health Review. 2020; 3(6): 17268-17280.

17. ORGANIZAÇÃO MUNDIAL DE SAÚDE (OMS). Annual Report 2001 for the ILAE/IBE/WHO Global Campaign against Epilepsy. Disponível em: http:www.who.int/mental_health/neurology/epilepsy/report_2019/en. Acessado em: 24 de março de 2021.

18. ORGANIZAÇÃO MUNDIAL DE SAÚDE (OMS). Mental health in primary care: illusion or inclusion World Health Organization. 2018. Disponível em: https://apps.who.int/iris/handle/10665/326298. Acessado em: 18 de outubro de 2021.

19. PÉREZ EB. Epilepsia e condições psiquiátricas relacionadas. Tratado de saúde mental da infância e adolescência da IACAPAP. cap.1.2. 2015, p. 3-7.

20. SCOTT AJ, et al. Anxiety and depressive disorders in people with epilepsy: A meta-analysis. Epilepsia. 2017; 58(6): 973-982.

21. SCOTT AJ, et al. How frequently is anxiety and depression identified and treated in hospital and community samples of adults with epilepsy? Epilepsy Behav. 2021; 115: 107703.

22. SCOTT RA, et al. Policy and Practice The treatment of epilepsy in developing countries: where do we go from here? World Health. 2001; 79(5): 344-51.

23. WALSH S, et al. Comorbid depression and associated factors in PNES versus epilepsy: Systematic review and metaanalysis. Seizure. 2018; 60: 44-56.

24. XIANGMIAO Q, et al. Antiepileptic effect of olanzapine in epilepsy patients with atypical depressive comorbidity. Epileptic Disorders. 2018; 20(3): 225-23. 